\title{
STONE'S THEOREM FOR A GROUP OF UNITARY OPERATORS OVER A HILBERT SPACE
}

\author{
HABIB SALEHI ${ }^{1}$
}

\begin{abstract}
The spectral representation for a group of unitary operators acting on a Hilbert space where the parameter set is a separable real Hilbert space is obtained. The usual spectral representation of such a group of unitary operators is when the parameter set is a locally compact abelian group (Stone's theorem). The main result used in the proof is the Bochner theorem on the representation of positive definite functions on a real Hilbert space.
\end{abstract}

Introduction. This paper discusses a spectral representation theorem for a group of unitary operators acting on a Hilbert space $H$, where the parameter set is a real Hilbert space $T$. The usual spectral representation of such a group of unitary operators (the well-known Stone theorem) is when the set $T$ is a locally compact abelian group [3, p. 392]. The main result used in the course of our work is the Bochner theorem on the representation of positive definite functions on a real Hilbert space $T$. This theorem is included in the interesting work of L. Gross [2] in Harmonic analysis on Hilbert space.

1. Preliminaries. We begin this section by introducing some notation which will be used later. $T$ will be a real Hilbert space. We will use $s, t$, etc. for points in $T$. By a Borel set in $T$ we shall mean a set in the $\sigma$-algebra determined by the collection of open sets in $T$. In $T$ the inner product and norm will be denoted by $(\cdot, \cdot)_{T}$ and $\left.\right|_{T}$. A complex-valued function $\varphi$ on $T$ will be called positive definite if for every finite set $t_{1}, \cdots, t_{n}$ of points in $T$ the matrix $\left(a_{i j}\right)$ defined by $a_{i j}=\varphi\left(t_{i}-t_{j}\right)$ is nonnegative definite. The following definition is due to L. Gross $[2$, p. 5].

1.1 Definition (TOPOLOGY $\tau$ FOR $T$ ). The topology of $\tau$ is defined as the weakest topology for which all Hilbert-Schmidt operators on $T$ into $T$ are continuous from $\tau$ to the strong topology of $T$.

Received by the editors April 26, 1971.

AMS 1970 subject classifications. Primary 43A35, 47D10; Secondary 60G25.

Key words and phrases. Group of unitary operators, spectral representation, Hilbert space, Hilbert-Schmidt operators, $\tau$-topology, positive definite, stationary stochastic processes.

${ }^{1}$ This work is supported by NSF GP-11626 and NSF GP-28658.

(c) American Mathematical Society 1972 
In these terms we can state Bochner's theorem as proved by L. Gross [2, p. 20], as follows:

1.2 THEOREM. A complex-valued function $\varphi$ on $T$ is positive definite and continuous with respect to the $\tau$-topology if and only if

$$
\varphi(t)=\int_{T} e^{i(t, s)} \mu(d s),
$$

where $\mu$ is a positive finite measure on $T$.

It is known that if $T$ is separable then $\varphi$ determines $\mu$ uniquely (cf. [2, p. 1 and p. 6]). Since a complex measure $\mu$ can be decomposed into $\mu=\mu_{1}-\mu_{2}+i\left(\mu_{3}-\mu_{4}\right)$, where $\mu_{1}, \mu_{2}, \mu_{3}, \mu_{4}$ are nonnegative finite measures, we can state the following corollary:

1.4 COROLlary. If $\psi$ is any complex-valued function defined on a separable real Hilbert space $T$ which has the form

$$
\psi(t)=\int_{T} e^{i(t, s)} d \mu,
$$

when $\mu$ is a complex-valued measure, then $\mu$ is uniquely determined by $\psi$.

The groups of unitary operators whose spectral representation we shall establish will be denoted by $\left\{U_{t}, t \in T\right\}$. Precisely speaking we have a complex Hilbert space $H$ \{with inner product $(\cdot, \cdot)_{H}$ and norm $|\cdot|_{H}$ \} and for each $t \in T$, we have a unitary operator $U_{t}$ acting on $H$ such that $U_{t} U_{s}=U_{t+s}$ for all $s, t \in T$. Points of $H$ will be denoted by $x, y$, etc. We shall assume in proving our spectral theorem that $U_{t}$ is weakly $\tau$-continuous in $t$, i.e., $\left(U_{t} x, y\right)$ is continuous with respect to the $\tau$-topology for each $x, y \in H$. We remark that our proof of Theorem 2 uses the representation of positive definite $\tau$-continuous functions $\varphi[1$, p. 20], in the same spirit as the corresponding Bochner theorem concerning positive definite functions on a locally compact abelian group was used to obtain the Stone's theorem for a group of unitary operators over a locally compact abelian group.

2. The spectral theorem. By a spectral measure on $T$ for a given Hilbert space $H$ we shall mean a family of operators $\{E(B)\}$ on $H$ into $H$ on $\mathscr{B}(T)$, the Borel sets in $T$ with the following properties:

(a) $E(B)$ is an orthogonal projection operator.

(b) $E(\varnothing)=0$.

(c) $E(T)=I$.

(d) If $B=\bigcup_{i} B_{i}$, when $B_{i}$ are disjoint sets in $\mathscr{B}(T)$, then $E(B)=$ $\sum_{i} E\left(B_{i}\right)$.

We can now state and prove our spectral theorem. 
2.2 THEOREM. Let $\left\{U_{t}, t \in T\right\}$ be a weakly $\tau$-continuous group of unitary operators on a given Hilbert space $H$, over a separable Hilbert space $T$. Then there exists a unique spectral measure $\{E(B), B \in \mathscr{B}(T)\}$ such that

$$
U_{t}=\int_{T} e^{i(t, s)} d E(s), \quad t \in T .
$$

$A$ bounded operator commutes with every $U_{t}$ if and only if it commutes with each $E(B), B \in \mathscr{B}(T)$.

Proof. For each $x \in H$, the function $\left(U_{t} x, x\right)$ is a positive definite function. Since by assumption $\left(U_{t} x, x\right)$ is $\tau$-continuous, by Theorem 1.2 and (1.3),

$$
\left(U_{t} x, x\right)=\int_{T} e^{i(t, s)} \mu_{x}(d s) .
$$

From (2.4) it follows that for each $x, y \in H$ there exists a complex-valued measure $\mu_{x, y}$ such that

$$
\left(U_{t} x, y\right)=\int_{T} e^{i(t, s)} \mu_{x, y}(d s) .
$$

The uniqueness of this representation (Corollary 1.4) then shows that for each $B \in \mathscr{B}(T), \mu_{x, y}(B)$ is a bilinear functional and also

$$
\mu_{x, y}(B)=\overline{\mu_{y, x}(B)} \text {. }
$$

We note that $\left|\mu_{x, y}(B)\right|^{2} \leqq \mu_{x, x}(B) \mu_{y, y}(B)$. Putting $t=0$ in (2.4) we get that

$$
\mu_{x, x}(B) \leqq|x|^{2}
$$

then (2.6) and (2.7) imply that $\mu_{x, y}(B)$ is a bounded bilinear functional on $H$. Hence by $[3$, p. 202], we conclude that for each Borel set $B \in \mathscr{B}(T)$ there is a bounded operator $E(B)$ on $H$ into $H$ such that $(E(B) x, y)=$ $\mu_{x, y}(B), E(B)=E^{*}(B)$. If $B_{1}, B_{2} \in \mathscr{B}(T)$ we have

$$
\begin{aligned}
\left(U_{t+s} x, y\right) & =\int_{T} e^{i(t+s . \xi)}(E(d \xi) x, y) \\
& =\int_{T} e^{i(t, \xi)} e^{i(t, \xi)}(E(d \xi) x, y)=\int e^{i(t, \xi)} v(d \xi),
\end{aligned}
$$

when $v(d \xi)=e^{i(s, \xi)}(E(d \xi) x, y)$. We also have

$$
\left(U_{t+s} x, y\right)=\int e^{i(t, \xi)}\left(E(d \xi) U_{s} x, y\right) .
$$

By the uniqueness result (Corollary 1.4), we have that, for each $B_{1} \in \mathscr{B}(T)$,

$$
\int e^{i(s, \xi)}\left(E\left((d \xi) \cap B_{1}\right) x, y\right)=\int_{B_{1}} e^{i(s, \xi)}(E(d \xi) x, y)=\left(E\left(B_{1}\right) U_{s} x, y\right) .
$$


Also we have

$$
\left(E\left(B_{1}\right) U_{s} x, y\right)=\left(U_{s} x, E\left(B_{1}\right) y\right)=\int e^{i(s, \xi)}\left(E(d \xi) x, E\left(B_{1}\right) y\right) .
$$

From (2.8) and (2.9) we obtain $\left(E\left(B \cap B_{1}\right) x, Y\right)=\left(E(B) x, E\left(B_{1}\right) Y\right)$. Therefore $E\left(B \cap B_{1}\right)=E(B) E\left(B_{1}\right)$. Since

$$
\left(U_{0} x, y\right)=(x, y)=\int \mu_{x, y}(d s)=\mu_{x, y}(T)
$$

we get that, for all $x, y \in H,(E(T) x, y)=(x, y)$; hence $E(T)=I$. Let $B=\bigcup B_{i}, B_{i}$ 's disjoint in $\mathscr{B}(T)$. Then

$$
(E(B) x, y)=\mu_{x, y}(B)=\mu_{x, y}\left(\bigcup B_{i}\right)=\sum_{i} \mu_{x, y}\left(B_{i}\right)=\sum_{i}\left(E\left(B_{i}\right) x, y\right) .
$$

Hence, $E(B)=\sum_{i} E\left(B_{i}\right)$.

This implies $E(\varnothing)=2 E(\varnothing)$ and hence $E(\varnothing)=0$. So that $E$ is a spectral measure. The existence of the integral $\int_{T} e^{i(t, \xi)} E(d \xi)$ which we call $V_{t}$ is well known. Then from the usual operational calculus it follows that $\left(V_{t} x, y\right)=\int e^{i(t, \xi)}(d E(w) x, y)$, so that if each $t \in T$ we have $\left(V_{t} x, y\right)=$ $\left(U_{t} x, y\right)$, and hence $V_{t}=U_{t}$.

In view of Corollary 1.4 the uniqueness of $E(\cdot)$ is clear.

Now let $V$ be any bounded linear operator on $H$ into $H$. An argument similar to the one given in $\left[1\right.$, p. 593] shows that $V$ commutes with $U_{t}$, $t \in T$, if and only if $V$ commutes with $E(B), B \in \mathscr{B}(T)$.

3. Application to stochastic processes. Let $(\Omega, B, P)$ be a probability space and let $\left\{X_{t}\right\}$ be a 2 nd order stochastic process with $t \in T\{T$ is a separable real Hilbert space $\}$. $\mathscr{E}$ will denote the expectation operator. If $\mathscr{E}\left(X_{t} \bar{X}_{s}\right)$ depends only on the difference of $t-s$ then $\left\{X_{t}\right\}$ is said to be a stationary stochastic process. The theory of such processes over any locally compact abelian group has been studied [4]. The same line of proof as in [4] can be used to prove:

3.1 THEOREM. (i) Let $\left\{X_{t}, t \in T\right\}$ be a stationary stochastic process with values in $L_{2}(\Omega, B, P)$.

(ii) Let $H=$ closed subspace in $L_{2}(\Omega, B, P)$ spanned by $\left\{X_{t}, t \in T\right\}$.

(iii) Let $\left\{X_{t}\right\}$ be $\tau$-continuous, i.e. the correlation function $\mathscr{E}\left(X_{t} \bar{X}_{0}\right)$ is continuous with respect to the $\tau$-topology of $T$.

Then

(a) There exists a unique spectral measure $E$ on $\mathscr{B}(T)$ for the Hilbert space $H$ such that

$$
X_{t}=\int_{T} e^{i(t, \xi)} E(d \xi) X_{0}, \quad \mathscr{E}\left(X_{t} \bar{X}_{s}\right)=\int_{T} e^{i(t-s, \xi)}\left(E(d \xi) X_{0}, X_{0}\right)
$$


The measure $d F=\left(E(d \xi) X_{0}, X_{0}\right)$ is called the spectral distribution of the process $\left\{X_{t}, t \in T\right\}$.

(b) The mapping $e^{i(t . \cdot)} \rightarrow X_{t}$ is an isometry on $L_{2, F}$ onto $H$.

Proof of (b) depends on the fact that $e^{i(t .)}$ are dense in $L_{2, F}(T)$ because of Corollary 1.4.

Remark. Theorem 3.1 and representation (3.2) can be used in the prediction theory of stationary stochastic processes over a separable Hilbert space to obtain results similar to the usual work in the prediction of stationary stochastic processes over a locally compact abelian group [4]. This work is being investigated by the author and the result will be published elsewhere.

\section{REFERENCES}

1. W. Ambrose, Spectral resolution of groups of unitary operators, Duke Math. J. 11 (1944), 589-595. MR 6, 131.

2. L. Gross, Harmonic analysis on Hilbert space, Mem. Amer. Math. Soc. No. 46 (1963). MR 28 \#4304.

3. F. Riesz and B. Sz.-Nagy, Leçons d'analyse fonctionnelle, 2nd ed., Akad. Kiadó, Budapest, 1953; English transl., Ungar, New York, 1955. MR 15, 132; MR 17, 175.

4. M. Rosenberg, Mutual subordination of multivariate stationary processes over any locally compact abelian group, Z. Wahrscheinlichkeitstheorie und Verw. Gebiete 12 (1969), 333-343. MR 40 \#5017.

Department of Mathematics, Michigan State University, East Lansing, MiCHIGAN 48823 